\title{
Neoadjuvant versus definitive chemoradiation in patients with squamous cell carcinoma of the esophagus
}

Stefan Münch ${ }^{1,2^{*}}$ (D) Steffi U. Pigorsch ${ }^{1}$, Michal Devečka ${ }^{1}$, Hendrik Dapper ${ }^{1}$, Marcus Feith ${ }^{3}$, Helmut Friess ${ }^{3}$, Wilko Weichert ${ }^{2,4}$, Moritz Jesinghaus ${ }^{4}$, Rickmer Braren ${ }^{5}$, Stephanie E. Combs ${ }^{1,2,6}$ and Daniel Habermehl ${ }^{1}$

\begin{abstract}
Background: Multimodal treatment with neoadjuvant chemoradiation followed by surgery $(n C R T+S)$ is the treatment of choice for patients with locally advanced or node-positive esophageal squamous cell carcinoma (E-SCC). Those who are unsuitable or who decline surgery can be treated with definitive chemoradiation (dCRT). This study compares the oncologic outcome of nCRT + S and dCRT in E-SCC patients.

Methods: Between 2011 and 2017, 95 patients with E-SCC were scheduled for dCRT or nCRT+ S with IMRT at our department. Patients undergoing dCRT received at least $50 \mathrm{~Gy}$ and those undergoing $\mathrm{nCRT}+\mathrm{S}$ received at least 41.4 Gy. All patients received simultaneous chemotherapy with either carboplatin and paclitaxel or cisplatin and 5-fluoruracil. We retrospectively compared baseline characteristics and oncologic outcome including overall survival (OS), progression-free survival (PFS) and site of failure between both treatment groups.

Results: Patients undergoing dCRT were less likely to have clinically suspected lymph node metastases (85\% vs. $100 \%, p=0.019$ ) than patients undergoing $\mathrm{nCRT}+\mathrm{S}$ and had more proximally located tumors (median distance from dental arch to cranial tumor border $20 \mathrm{~cm}$ vs. $26 \mathrm{~cm}, p<0.001$ ). After a median follow up of 25.6 months for surviving patients, no significant differences for OS and PFS were noticed comparing nCRT + $S$ and dCRT. However, the rate of local tumor recurrence was significantly higher in patients treated with dCRT than in those treated with $\mathrm{nCRT}+\mathrm{S}(38 \%$ vs. $10 \%, p=0.002)$. Within a multivariate Cox regression model, age, tumor location, and tumor grading were the only independent parameters affecting OS and PFS. In addition to that, proximal tumor location was the only parameter independently associated with an increased risk for local treatment failure.

Conclusion: In E-SCC patients treated with either dCRT or nCRT + S, a higher rate of local tumor recurrence was seen in patients treated with dCRT than in patients treated with nCRT $+\mathrm{S}$. There was at least a trend towards an improved OS and PFS in patients undergoing nCRT + S. However, this should be interpreted with caution, because proximal tumor location was the only parameter independently affecting the risk of local tumor recurrence.
\end{abstract}

Keywords: Squamous cell carcinoma of the esophagus, Definitive chemoradiation, Neoadjuvant chemoradiation, Modern radiation techniques

\footnotetext{
* Correspondence: stefan.muench@mri.tum.de

${ }^{1}$ Department of Radiation Oncology, Klinikum rechts der Isar, Technical

University Munich, Ismaninger Str. 22, 81675 Munich, Germany

${ }^{2}$ German Cancer Consortium (DKTK), Partner Site Munich, Munich, Germany

Full list of author information is available at the end of the article
}

(c) The Author(s). 2019 Open Access This article is distributed under the terms of the Creative Commons Attribution 4.0 International License (http://creativecommons.org/licenses/by/4.0/), which permits unrestricted use, distribution, and reproduction in any medium, provided you give appropriate credit to the original author(s) and the source, provide a link to the Creative Commons license, and indicate if changes were made. The Creative Commons Public Domain Dedication waiver (http://creativecommons.org/publicdomain/zero/1.0/) applies to the data made available in this article, unless otherwise stated. 


\section{Background}

In patients with locally advanced esophageal squamous cell carcinoma (E-SCC) trimodal therapy including neoadjuvant chemoradiation followed by surgery $(n C R T+S)$ demonstrated its potential to improve overall survival (OS) and disease-free survival (DFS) compared to surgery alone [1-3]. Therefore, it has been established as the treatment of choice for patients with non-cervical E-SCC who are suitable for surgery [4]. In contrast, patients with locally advanced E-SCC who decline surgery, are medically inoperable or have unresectable tumors should undergo definitive chemoradiation (dCRT). Because of this, patients undergoing dCRT, show in general more advanced tumors and are in a worse general condition than patients who are treated with $\mathrm{nCRT}+\mathrm{S}$. Therefore, a meaningful comparison of both treatment options is difficult. Up to the present there are only two randomized trials comparing $\mathrm{dCRT}$ and $\mathrm{nCRT}+\mathrm{S}$ in patients with esophageal cancer (EC) $[5,6]$. The study by Stahl and colleagues, which was terminated early, revealed no significant difference for OS between both treatment groups, while $\mathrm{nCRT}+\mathrm{S}$ was associated with a significantly increased local progression-free survival [5]. However, all patients in this trial received induction chemotherapy, which is not in line with current treatment recommendations and therefore compromises the interpretation of the results. In accordance with that, a French study also found no difference in OS between surgery or continuation of chemoradiation (CRT) in patients with good response to CRT [6]. Furthermore, there was no significant difference regarding local control rate. One of the major problems of this study is that only patients with good response to CRT were randomized to continuation of chemoradiation or surgery. Therefore, the results are only applicable to a specific subgroup of patients. These results are in line with a recently published retrospective trial by Haefner et al. [7] that compared nCRT $+\mathrm{S}$ and dCRT in patients with locally advanced EC. After a median follow-up time of 20.4 months, no significant differences were seen regarding progression-free survival (PFS) and OS between both treatment regimes. In contrast, a recent retrospective cohort study by Barbetta et al. [8] demonstrated an improved OS and DFS after nCRT $+\mathrm{S}$ compared to dCRT in patients with thoracic or distal E-SCC. In addition, further retrospective trials also demonstrated an increased survival after nCRT + S in EC patients [9-11]. One major problem with the studies mentioned above is, that most of them included patients with both, adenocarcinoma (AC) and E-SCC. This clearly affects the results because the effect of CRT is higher for patients with E-SCC than for patients with adenocarcinoma [2] and in general these two tumor types - although occurring at the same location - are biology-wise completely different tumor entities. Moreover, many patients were treated with 3-dimensional conformal radiotherapy (3D-CRT). Although no significant differences regarding progression-free survival (PFS) and OS were seen in two retrospective trials comparing 3D-CRT and modern radiation techniques like intensity-modulated radiotherapy (IMRT) for $n C R T+S$ or dCRT in patients with EC $[12,13]$, the use of modern radiation techniques is at least associated with lower doses to the organs at risk in patients undergoing nCRT $+\mathrm{S}$ [12]. Due to the incoherent results of previous trials, our aim is to report the results for patients with only E-SCC, who underwent $\mathrm{dCRT}$ or $\mathrm{nCRT}+\mathrm{S}$ with modern radiation techniques.

\section{Methods}

This study includes 95 patients with E-SCC who were treated with either dCRT or nCRT $+\mathrm{S}$ at our department between 2011 and 2017. Inclusion criteria were histologically proven E-SCC, curative treatment approach with either dCRT or nCRT + S, simultaneous chemotherapy, the use of IMRT and at least one follow-up after the end of therapy. Exclusion criteria were distant metastases (M1) at the time of diagnosis, simultaneous radiotherapy or CRT of a second cancer, discontinuation of therapy due to any reason and a total radiation dose <50 Gray (Gy) for patients undergoing dCRT or $<41.4 \mathrm{~Gy}$ for patients undergoing $\mathrm{nCRT}+\mathrm{S}$. All patients were staged with ${ }^{18}$ Fludeoxyglucose positron emission tomography combined with computed tomography $\left({ }^{18} \mathrm{FDG}-\mathrm{PET} / \mathrm{CT}\right) \quad(95 \%)$ or computed tomography alone (5\%). Whenever possible, endoscopic ultrasound was also used to assess T- and $\mathrm{N}$-stage.

\section{Treatment}

A total of 40 patients were treated with $\mathrm{nCRT}+\mathrm{S}$ and 55 patients were treated with dCRT. Thereby, dCRT was most commonly used for patients with cervical tumors (47\%), patients who refused surgery (27\%) and patients who were inoperable due to underlying internistic medical conditions (11\%). Additionally, irresectable tumors or high age of patients with reduced general condition were the reasons for choosing dCRT in $7 \%$ and $7 \%$ of patients, respectively. Treatment planning was based on planning computed tomography $(\mathrm{CT})$ in supine position. All available diagnostic information (esophagogastroduodenoscopy with or without endoscopic ultrasound, ${ }^{18}$ FDG-PET, and CT scans) were used to identify the gross tumor volume (GTV), defined as the macroscopic primary tumor and all putative lymph node metastases. For the planning target volume, a longitudinal safety margin of $4-5 \mathrm{~cm}$ and a radial safety margin of $1.5-2 \mathrm{~cm}$ were added to the GTV. Due to the long time interval in which patients were included for this analysis and the lack of guidelines regarding elective inclusion of regional 
lymphatic pathways into the clinical target volume, there was no standardized regional lymphatic coverage policy. In general, the periesophageal and mediastinal lymphatics were at least partially covered by the axial safety margin around the primary tumor. Further coverage of periesophageal and mediastinal lymphatics was done on an individual base, depending on the individual expertise of the treating radiation oncologist. However, additional inclusion of the cervical or abdominal/ coeliac lymphatics was seen in $68 \%$ of patients undergoing nCRT + $\mathrm{S}$ before 2014 and no patient undergoing $\mathrm{nCRT}+\mathrm{S}$ after 2014. This difference is caused by the fact, that since 2014 patients were treated analogously to the CROSS-Trial. In patients treated with dCRT, elective nodal irradiation (cervical and/or abdominal/coeliac) was done in $65 \%$ of patients. In all patients, irradiation was applied using 6-/or $15 \mathrm{MeV}$ photons delivered with IMRT. Median total radiation dose was 41.4 Gy (range 41.4-45 Gy) for patients treated with nCRT $+\mathrm{S}$ and 54 Gy (range 50-64.8 Gy) for patients treated with dCRT, respectively.

In patients undergoing $\mathrm{nCRT}+\mathrm{S}$, median radiation dose was $43.2 \mathrm{~Gy}$ for patients with cervical tumor location and $41.4 \mathrm{~Gy}$ for patients with thoracic or abdominal tumor location. In those undergoing dCRT, median radiation dose was $54 \mathrm{~Gy}$ in patients with cervical tumor location, $56 \mathrm{~Gy}$ in patients with thoracic tumor location and $55.8 \mathrm{~Gy}$ in patients with abdominal tumor location. Thirty-nine patients (98\%) who underwent nCRT $+\mathrm{S}$ received simultaneous chemotherapy with either carboplatin and paclitaxel or cisplatin and 5-fluoruracil. One patient received simultaneous chemotherapy with only cisplatin. Of patients who underwent dCRT, 48 (87\%) received simultaneous chemotherapy with either carboplatin and paclitaxel or cisplatin and 5-fluoruracil (5FU), while one patient $(2 \%)$ received simultaneous chemotherapy with carboplatin and 5FU, one patient (2\%) received carboplatin only, two patients (4\%) received cisplatin only and 3 patients (5\%) received only fluoropyrimidine-based chemotherapy.

In patients who underwent nCRT $+S$, the median time interval between neoadjuvant chemoradiation and surgery was 42 days (range 25-86 days) and complete tumor resection was achieved in $97 \%$ of patients. Histopathologic tumor response to neoadjuvant treatment was assessed according to the classification published by Becker et al. [14]. Thereby, complete tumor response, less than $10 \%$ vital tumor, $10-50 \%$ vital tumor and more than $>50 \%$ vital tumor was seen in $38 \%, 43 \%, 7 \%$ and $12 \%$ of patients, respectively. Regarding post-surgical morbidity, anastomotic insufficiency was seen in $9 \%$ of patients after nCRT + S. No cause of death was seen within the first four weeks after treatment $(\mathrm{dCRT}$ or $\mathrm{nCRT}+\mathrm{S})$.

\section{Follow-up}

After completion of treatment, all patients were regularly invited to follow-up examinations according to our institutional standard. The first follow-up was scheduled approximately 6-8 week after treatment and included clinical examinations, esophagogastroduodenoscopy, and thoracic computed tomography, thereafter in 3-months intervals or as needed clinically.

\section{Statistics}

Comparison of nominally scaled baseline parameters was done using chi-square test. For ordinally scaled parameters, the Fishers-exact-test was used to compare treatment groups, while Mann-Whitney-U-test was used for interval scaled variables. OS was defined as time between the beginning of treatment and death. Patients lost to follow-up were censored. PFS was defined as the period of time between beginning of treatment and any proven tumor recurrence or death for any reason. Overall survival and progression-free survival where compared using the log-rank test. To analyze the effect of different parameters on OS, PFS and local, regional or distant tumor recurrence we also performed univariate and multivariate Cox regression analyses. All statistical tests were conducted in an exploratory manner on two-sided 5\% significance levels using the software SPSS Statistics 18 version 18.0.0 (IBM SPSS Statistics, Armonk, U. S.).

\section{Results}

\section{Baseline characteristics}

Patients' baseline clinical data and tumor parameters can be seen in Table 1. Within the dCRT group, patients were slightly older ( 68 years vs. 65 years) and the rate of male patients $(73 \%$ vs. $55 \%)$ was higher than in the group of patients treated with nCRT $+S$, but these differences were not statistically significant. In both treatment groups most patients had T3 tumors with moderate or poor tumor cell differentiation (G2/G3). In addition, median tumor length was $5 \mathrm{~cm}$ in both groups. The rate of clinically suspected pretherapeutic lymph node metastases was higher in patients undergoing $\mathrm{nCRT}+\mathrm{S}$ than in patients undergoing dCRT $(100 \%$ vs. $85 \%, p=0.019$ ). Tumor location was classified according to the position of the tumor as it was seen in the esophagogastroduodenoscopy (EGD). If the oral tumor margin was seen within the first $3 \mathrm{~cm}$ of the esophagus, the tumor was classified as cervical. If the center of the tumor was not more than $3 \mathrm{~cm}$ away from the cardia, the tumor was classified as an abdominal tumor. All other tumors were classified as thoracic. While there was a comparable rate of patients with abdominal tumor location in both treatment groups, a higher rate of 
Table 1 Patients' baseline and tumor parameters

\begin{tabular}{|c|c|c|c|}
\hline Parameter & $\begin{array}{l}\mathrm{nCRT}+\mathrm{S} \\
(n=40)\end{array}$ & $\begin{array}{l}\text { dCRT } \\
(n=55)\end{array}$ & $p$-value \\
\hline \multicolumn{4}{|l|}{ Age, (years) } \\
\hline Median & 65 & 68 & \multirow[t]{2}{*}{0.079} \\
\hline IQR & $56-72$ & $62-74$ & \\
\hline Male sex & $22(55 \%)$ & $40(73 \%)$ & 0.085 \\
\hline \multicolumn{4}{|l|}{ T-stage (cT) } \\
\hline Tis & $0(0 \%)$ & $1(2 \%)$ & \multirow[t]{5}{*}{0.148} \\
\hline $\mathrm{T} 1$ & $1(3 \%)$ & $3(5 \%)$ & \\
\hline $\mathrm{T} 2$ & $7(18 \%)$ & $8(15 \%)$ & \\
\hline T3 & $32(80 \%)$ & 37 (67\%) & \\
\hline T4 & $0(0 \%)$ & $6(11 \%)$ & \\
\hline \multicolumn{4}{|l|}{$\mathrm{N}$-stage $(\mathrm{cN})$} \\
\hline $\mathrm{N}+$ & $40(100 \%)$ & 47 (85\%) & 0.019 \\
\hline \multicolumn{4}{|l|}{ Grading } \\
\hline G1 & $1(3 \%)$ & $0(0 \%)$ & \multirow[t]{3}{*}{0.729} \\
\hline $\mathrm{G} 2$ & $20(53 \%)$ & $26(53 \%)$ & \\
\hline G3 & 17 (45\%) & $23(47 \%)$ & \\
\hline \multicolumn{4}{|l|}{ Tumor length, (cm) } \\
\hline Median & 5 & 5 & \multirow[t]{2}{*}{0.445} \\
\hline IQR & $3-7$ & $4-7$ & \\
\hline \multicolumn{4}{|c|}{ Distance from dental arch to cranial tumor border, $(\mathrm{cm})$} \\
\hline Median & 26 & 20 & \multirow[t]{2}{*}{$<0.001$} \\
\hline IQR & $24-30$ & $17-26$ & \\
\hline \multicolumn{4}{|l|}{ Tumor location } \\
\hline Cervical & $4(10 \%)$ & $26(47 \%)$ & \multirow[t]{3}{*}{$<0.001$} \\
\hline Thoracic & $35(88 \%)$ & $28(51 \%)$ & \\
\hline Abdominal & $1(3 \%)$ & $1(2 \%)$ & \\
\hline \multicolumn{4}{|l|}{ Cumulative RT dose, (Gy) } \\
\hline Median & 41.4 & 54 & \multirow[t]{2}{*}{$<0.001$} \\
\hline IQR & $41.4-45$ & $54-59.4$ & \\
\hline Complete tumor resection & $36(97 \%)$ & - & - \\
\hline
\end{tabular}

IQR inter-quartiles-range, Gy Gray, $R T$ radiotherapy

cervical tumor location $(47 \%$ vs. $10 \%)$ was seen in patients treated with dCRT.

\section{Survival}

After a median follow-up of 25.6 months for surviving patients, median OS was 43.3 months for patients undergoing $\mathrm{nCRT}+\mathrm{S}$ and 23.2 months for patients undergoing dCRT $(p=0.228)$. 1y-OS, $2 \mathrm{y}$-OS and $3 \mathrm{y}-\mathrm{OS}$ was $76.6 \%, 65.0 \%$ and $57.2 \%(\mathrm{nCRT}+\mathrm{S})$ and $72.6 \%, 49.3 \%$ and $38.6 \%$ (dCRT), respectively (Fig. 1).

No significant difference was seen after exclusion of patients with cervical tumor location. After a median follow-up of 28.9 months for surviving patients, median OS was 43.3 months for patients undergoing
nCRT $+\mathrm{S}$ and 20.8 months for patients undergoing dCRT $(p=0.211)$ (Table 2$)$.

Median PFS was 18.3 and 12.7 months in patients treated with $\mathrm{nCRT}+\mathrm{S}$ and dCRT, respectively (Fig. 2, $p=0.108)$. Corresponding 1y-PFS, $2 \mathrm{y}$-PFS and $3 y$-PFS were $67.6 \%, 42.6 \%$ and $42.6 \%$ in patients undergoing $\mathrm{nCRT}+\mathrm{S}$ and $51.0 \%, 29.9 \%$ and $26.6 \%$ in patients undergoing dCRT.

After exclusion of patients with cervical tumors, median PFS was 19.3 months for patients treated with $\mathrm{nCRT}+\mathrm{S}$ and 14.0 months for patients treated with dCRT $(p=0.231)$ (Table 2).

\section{Treatment failure}

In summary, local or regional treatment failure was seen in $23 \%$ of patients, who were treated with nCRT $+\mathrm{S}$ and in $40 \%$ of patients, who were treated with dCRT ( $p=$ 0.081). Table 2 demonstrates patterns of failure for patients treated with $\mathrm{dCRT}$ or $\mathrm{nCRT}+\mathrm{S}$. While no significant difference was seen for the rate of regional treatment failure $(23 \%(\mathrm{nCRT}+\mathrm{S})$ vs. $13 \%(\mathrm{dCRT}), p=$ 0.269 ), dCRT was associated with an increased risk of local recurrence $(10 \%(\mathrm{nCRT}+\mathrm{S})$ vs. $38 \%(\mathrm{dCRT})$, $p=0.002)$. Distant treatment failure occurred in $10 \%$ $(\mathrm{nCRT}+\mathrm{S})$ and $16 \%(\mathrm{dCRT})$ of patients, respectively $(p=0.547)$. In patients with treatment failure, the first site of recurrence was local/regional, distant or both in $70 \%, 10 \%$ and $20 \%$ in patients treated with nCRT $+\mathrm{S}$ and $71 \%, 25 \%$ and $4 \%$ in patients treated with $\mathrm{dCRT} \quad(p=0.115)$. Regarding failure pattern, out-of-field locoregional recurrence was seen in 2 patients (13\%). Thereby, one patient underwent nCRT + $\mathrm{S}$ without elective nodal irradiation, while the other patient underwent dCRT. Elective nodal irradiation was done at the height of the primary tumor and the longitudinal safety margins in this patient.

When excluding patients with cervical tumor location, there was still a trend towards an increased rate of local tumor recurrence in patients undergoing dCRT (28\% vs. $8 \%$; $p=0.051)$. Comparable to the results for the whole cohort, no significant difference was seen for the rate of regional tumor recurrence (10\% vs. $19 \%, p=0.491)$ or distant treatment failure $(14 \%$ vs. $8 \%, p=0.691$ ) between both groups (Table 2 ).

After treatment failure, $15 \%$ of patients received local salvage treatment with either surgery or radio(chemo)therapy, and $59 \%$ of patients underwent systemic chemotherapy with palliative intent. $27 \%$ of patients received no further specific treatment, but only best-supportive care.

Results of univariate and multivariate Cox regression analyses are demonstrated in Table 3 and Table 4 . In the univariate Cox regression model, treatment regimen (nCRT + S vs. dCRT) significantly affected the risk of local recurrence, but not OS or PFS. Grading was the 


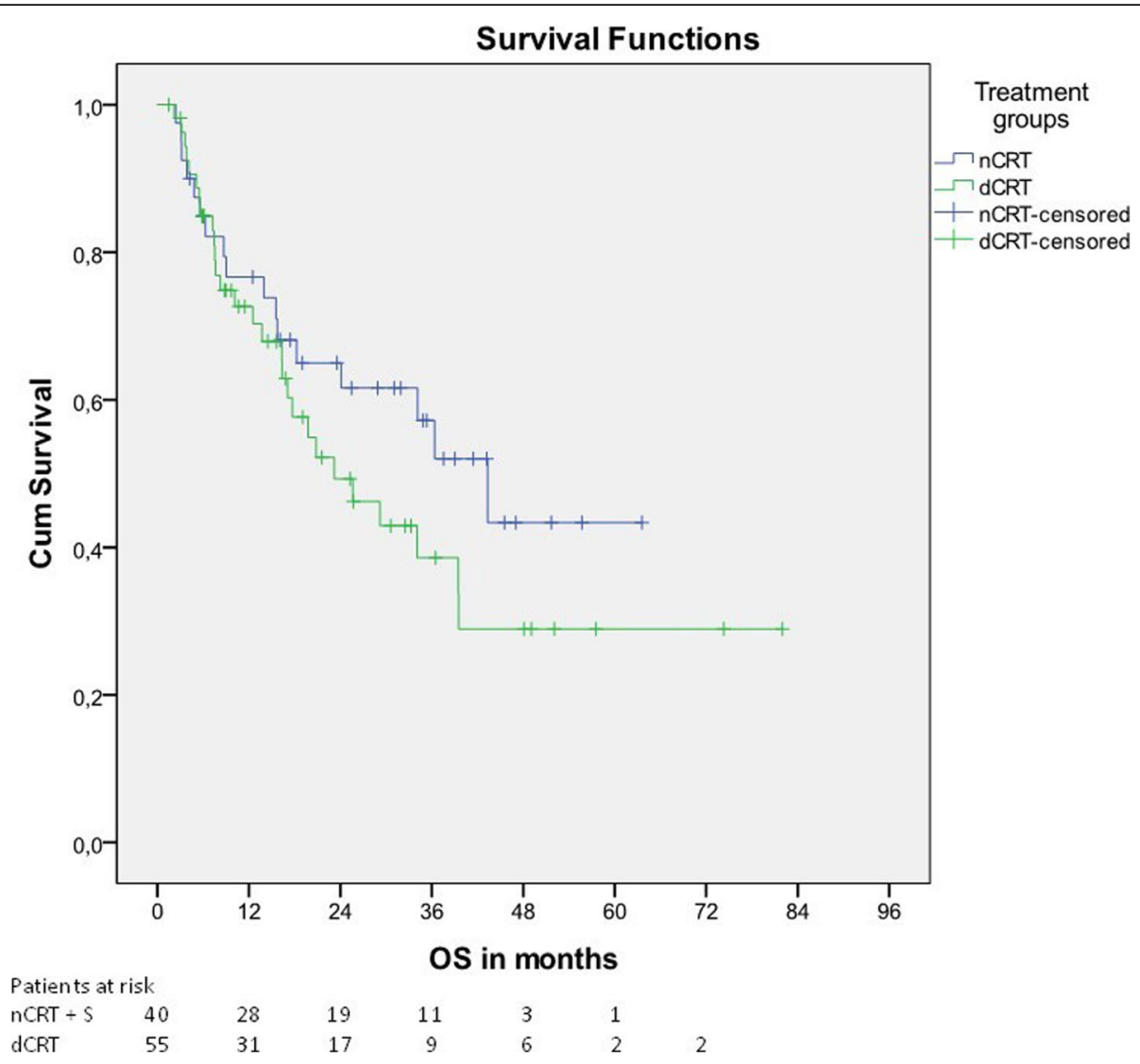

Fig. 1 Overall survival

only parameter significantly affecting OS, while tumor location significantly affected PFS and risk of local tumor recurrence. However, in the multivariate model, treatment regime did not significantly affect OS, PFS or local recurrence. Increasing age and good tumor differentiation (G1/2) were associated with worse OS (Hazard ratio (HR) 1.064, 95\% confidence interval (95\% CI) 1.019-1.110, $p=0.005$ (age); Hazard ratio (HR) 2.674, 95\% confidence interval (95\% CI) 1.299-4.503, $p=0.008$ (G1/2 vs. G3)) and PFS (HR 1.054, 95\% CI 1.016-1.093, $p=0.005$ (age); Hazard ratio (HR) 2.034, 95\% confidence interval (95\% CI) $1.098-3.768, p=0.024$ (G1/2 vs. G3)), while an increasing distance of the dental arch to the cranial tumor border was associated with increased OS (HR 0.939, 95\% CI 0.884-0.998, $p=0.043$ ), increased PFS (HR 0.925, 95\% CI 0.876-0.976, $p=0.005$ ) and a lower rate of local recurrence (HR 0.843, 95\% CI 0.760$0.935, p=0.001)$.

\section{Discussion}

In this analysis, we compared outcome of nCRT $+\mathrm{S}$ and dCRT in patients with E-SCC. Thereby, we only included patients who were treated with IMRT, to represent current clinical practice. While no significant

Table 2 Oncologic outcome for the whole cohort and for patients with thoracic or abdominal tumor location only

\begin{tabular}{|c|c|c|c|c|c|c|}
\hline \multirow[t]{2}{*}{ Oncologic Outcome } & \multicolumn{3}{|c|}{ All patients $(n=95)$} & \multicolumn{3}{|c|}{ Patients with thoracic or abdominal tumor location only $(n=65)$} \\
\hline & $\begin{array}{l}\mathrm{nCRT}+\mathrm{S} \\
(n=40)\end{array}$ & $\begin{array}{l}\mathrm{dCRT} \\
(n=55)\end{array}$ & $p$-value & $\begin{array}{l}\mathrm{nCRT}+\mathrm{S} \\
(n=36)\end{array}$ & $\begin{array}{l}d \text { dCRT } \\
(n=29)\end{array}$ & $p$-value \\
\hline Overall survival in months (median) & 43.3 & 23.2 & 0.228 & 43.3 & 20.8 & 0.211 \\
\hline Progression-free survival in months (median) & 18.3 & 12.7 & 0.108 & 19.3 & 14.0 & 0.115 \\
\hline \multicolumn{7}{|l|}{ Patterns of treatment failure } \\
\hline Local failure, n (\%) & $4(10 \%)$ & $21(38 \%)$ & 0.002 & $3(8 \%)$ & $8(28 \%)$ & 0.051 \\
\hline Regional failure, $\mathrm{n}(\%)$ & $9(23 \%)$ & $7(13 \%)$ & 0.269 & $7(19 \%)$ & $3(10 \%)$ & 0.491 \\
\hline Distant failure, n (\%) & $4(10 \%)$ & $9(16 \%)$ & 0.547 & $3(8 \%)$ & $4(14 \%)$ & 0.691 \\
\hline
\end{tabular}




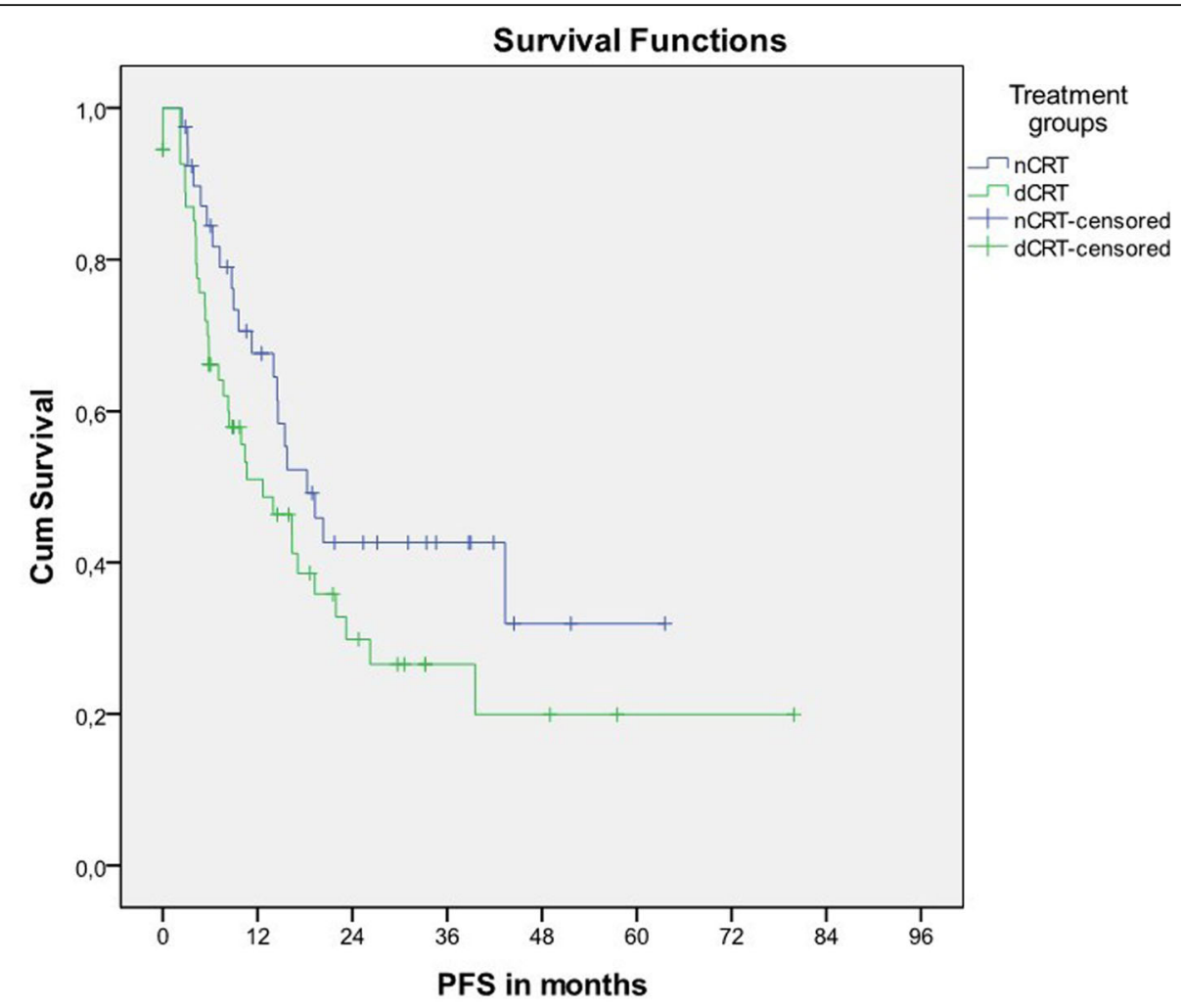

Patients at risk

$\begin{array}{llllllll}\mathrm{nCRT}+\mathrm{S} & 40 & 23 & 12 & 7 & 2 & 1 & \\ \mathrm{dCRT} & 55 & 22 & 10 & 4 & 3 & 1 & 1\end{array}$

Fig. 2 Progression-free survival

differences were seen for OS and PFS, the rate of local tumor recurrence was significantly higher in patients treated with dCRT than in those treated with nCRT $+\mathrm{S}$. However, in a multivariate Cox regression analysis, treatment regime was not independently associated with OS, PFS or rate of local tumor recurrence. Instead, the only parameter independently affecting OS, PFS, and rate of local tumor recurrence was tumor location, while patients' age and tumor grade were independently associated with OS and PFS.
While our absolute data for OS and PFS are comparable with two other recent studies $[7,8]$, there are conflicting results in terms of the relative difference between patients treated with nCRT $+\mathrm{S}$ and patients treated with dCRT. Haefner and colleagues [7] compared dCRT with nCRT $+\mathrm{S}$ in patients with esophageal cancer. In contrast to our study, the authors included patients with $\mathrm{AC}$ and patients treated with dCRT received two cycles of adjuvant chemotherapy with cisplatin and 5-fluorouracil.

Table 3 Univariate Cox regression analysis

\begin{tabular}{|c|c|c|c|c|c|c|}
\hline Parameter & $\begin{array}{l}\text { OS HR } \\
{[95 \% \mathrm{Cl}]}\end{array}$ & $p$-value & $\begin{array}{l}\text { PFS HR } \\
{[95 \% \mathrm{CI}]}\end{array}$ & $p$-value & $\begin{array}{l}\text { LR HR } \\
{[95 \% \mathrm{Cl}]}\end{array}$ & $p$-value \\
\hline Treatment Regimen (nCRT + S vs. dCRT) & $0.690[0.377 ; 1.264]$ & 0.230 & $0.643[0.374 ; 1.107]$ & 0.111 & $0.199[0.068 ; 0.582]$ & 0.003 \\
\hline Age (continuous) & $1.025[0.990 ; 1.061]$ & 0.160 & $1.025[0.994 ; 1.057]$ & 0.115 & $1.009[0.966 ; 1.053]$ & 0.699 \\
\hline Sex (female vs. male) & $0.830[0.446 ; 1.545]$ & 0.557 & $0.807[0.462 ; 1.410]$ & 0.451 & $0.775[0.334 ; 1.80]$ & 0.553 \\
\hline Tumor lengths, cm (continuous) & $0.996[0.899 ; 1.104]$ & 0.946 & $1.012[0.925 ; 1.107]$ & 0.798 & $1.022[0.895 ; 1.167]$ & 0.744 \\
\hline Tumor location (continuous) & $0.954[0.907 ; 1.004]$ & 0.069 & $0.940[0.897 ; 0.985]$ & 0.010 & $0.844[0.773 ; 0.921]$ & $<0.001$ \\
\hline T-stage (Tis/T1/2 vs. T3/4) & $1.295[0.618 ; 2.714]$ & 0.493 & $0.966[0.485 ; 1.924]$ & 0.921 & $1.319[0.524 ; 3.316]$ & 0.557 \\
\hline N-stage (N0 vs N1) & $1.780[0.965 ; 4.560]$ & 0.230 & $1.167[0.464 ; 2.935]$ & 0.743 & $1.207[0.284 ; 5.136]$ & 0.799 \\
\hline Grading (G1/2 vs. G3) & $2.059[1.084 ; 3.911]$ & 0.027 & $1.673[0.961 ; 2.912]$ & 0.069 & $2.065[0.882 ; 4.837]$ & 0.095 \\
\hline
\end{tabular}


Table 4 Multivariate Cox regression analysis

\begin{tabular}{|c|c|c|c|c|c|c|}
\hline Parameter & $\begin{array}{l}\text { OS HR } \\
{[95 \% \mathrm{Cl}]}\end{array}$ & $p$-value & $\begin{array}{l}\text { PFS HR } \\
{[95 \% \mathrm{Cl}]}\end{array}$ & $p$-value & $\begin{array}{l}\text { LR HR } \\
{[95 \% \mathrm{Cl}]}\end{array}$ & $p$-value \\
\hline Treatment Regimen (nCRT + S vs. dCRT) & $1.453[0.688 ; 3.070]$ & 0.327 & $1.135[0.592 ; 2.175]$ & 0.704 & $0.434[0.136 ; 1.382]$ & 0.158 \\
\hline Age (continuous) & $1.064[1.019 ; 1.110]$ & 0.005 & $1.054[1.016 ; 1.093]$ & 0.005 & $1.041[0.984 ; 1.101]$ & 0.165 \\
\hline Sex (female vs. male) & $0.634[0.316 ; 1.273]$ & 0.200 & $0.696[0.370 ; 1.311]$ & 0.263 & $0.763[0.277 ; 2.100]$ & 0.601 \\
\hline Tumor lengths, cm (continuous) & $1.044[0.933 ; 1.168]$ & 0.451 & $1.033[0.932 ; 1.145]$ & 0.541 & $1.057[0.912 ; 1.224]$ & 0.462 \\
\hline Tumor location (continuous) & $0.939[0.884 ; 0.998]$ & 0.043 & $0.925[0.876 ; 0.976]$ & 0.005 & $0.843[0.760 ; 0.935]$ & 0.001 \\
\hline T-stage (Tis/T1/2 vs. T3/4) & $1.434[0.639 ; 3.221]$ & 0.383 & $1.118[0.527 ; 2.369]$ & 0.772 & $1.997[0.665 ; 5.993]$ & 0.217 \\
\hline N-stage (N0 vs N1) & $1.556[0.558 ; 4.337]$ & 0.398 & $0.821[0.302 ; 2.234]$ & 0.699 & $0.605[0.135 ; 2.702]$ & 0.510 \\
\hline Grading (G1/2 vs. G3) & $2.674[1.299 ; 4.503]$ & 0.008 & 2.034 [1.098; 3.768] & 0.024 & $2.322[0.835 ; 6.453]$ & 0.106 \\
\hline
\end{tabular}

$I Q R$ inter-quartiles-range, OS overall survival, PFS progression-free survival, $L R$ local recurrence, $H R$ Hazard ratio, $C I$ Confidence interval

After a median follow-up of 20.4 months, no significant differences were visible for median OS (25.9 months vs. 20.6 months) and PFS (14.9 months vs. 15.6 months). In contrast to that, Barbetta and colleagues [8] reported an improved OS (median OS 2.3 years vs. 3.1 years) and DFS (median DFS 1 year vs. 1.8 years) after nCRT + S. The most obvious difference to the present study is the exclusion of patients with cervical or upper thoracic tumors. In our study, a more proximal tumor location was associated with shorter OS and PFS within multivariate Cox regression analysis. While we found no data evaluating the impact of tumor location after nCRT $+\mathrm{S}$ or dCRT in E-SCC patients, proximal tumor location was associated with decreased OS in patients with pT2-3N0M0 carcinoma after surgery alone [15]. As patients with cervical tumor location are typically treated with dCRT [16], and therefore the rate of patients with cervical tumor location is frequently higher within the subgroup of patients treated with dCRT, this might explain the improved OS after $\mathrm{nCRT}+\mathrm{S}$ in some trials. Interestingly, the only study that excluded patients with cervical or upper thoracic tumor location and performed a propensity score-matched analysis, also reported an improved OS for E-SCC patients undergoing nCRT $+\mathrm{S}$ [8]. One can speculate that especially after exclusion of patients with cervical or upper thoracic tumor location the number of patients in most trials would be too low to reveal any significant differences in OS. Another factor which might affect the results is the fact that patients who underwent dCRT in our study had a significantly lower rate of lymph node metastases. This might impact the results since it is well established that lymph node involvement is an important and independent prognostic factor in EC patients [17-19].

In accordance with other studies $[5,8,10]$, dCRT was associated with an increased rate of local tumor recurrence in our study. However, Stahl [5] and Liao [10] included patients with different tumor locations including patients with tumors of the cervical esophagus. Based on our results, it is conceivable that the difference in the rate of local tumor recurrence in these studies is biased by differences regarding tumor locations between both cohorts. Although, after excluding patients with cervical tumors, we still recognized a strong trend towards an increased local tumor control after nCRT $+\mathrm{S}$ compared to $\operatorname{dCRT}(p=0.051)$. In addition, in a recent trial by Barbetta and colleagues [8], which also demonstrated an increased risk of local tumor recurrence after dCRT compared to nCRT $+S$, this kind of bias was ruled out by performing a 1:1 propensity score-matching approach and excluding patients with tumors of the upper esophagus.

In accordance with the studies by Haefner et al. [7] and Barbetta et al. [8], the most common reason for treatment failure in patients undergoing dCRT was local or regional tumor recurrence. The absolute rate of local and regional recurrences after dCRT in our study was slightly higher than in the study by Haefner and colleagues (local $38 \%$ vs. $24 \%$; regional $13 \%$ vs. $4 \%$ ). This difference might be explained by the fact, that patients treated with dCRT were were more likely to have tumors of the cervical esophagus (47\% vs. $16 \%$ ) and were also more likely to have lymph node metastases $(85 \%$ vs. $77 \%)$. Interestingly, our results for local and regional tumor recurrence are comparable to the results by Barbetta and colleagues, despite patients with upper esophageal carcinoma were excluded in their study. The higher rate of regional tumor recurrence in patients undergoing $\mathrm{nCRT}+\mathrm{S}$ in the study by Barbetta et al., might be explained by the higher rate of patients with lymph node metastases (100\% vs. $85 \%$ ), which is an independent risk factor for both, locoregional and distant recurrence after nCRT $+\mathrm{S}$ [20]. Therefore, the difference in terms of the rate of lymph node metastases might also explain the higher rate of regional recurrence after $\mathrm{nCRT}+\mathrm{S}$ in our study compared to the results by Haefner et al. (23\% vs. $5 \%$ ) [7].

For both, patients treated with nCRT $+\mathrm{S}$ and patients treated with $\mathrm{dCRT}$, the rate of distant disease recurrence 
in our study is remarkably lower than in other recent trials $[7,8]$. While the exclusion of patients with $\mathrm{AC}$, who have a significantly higher risk of distant tumor recurrence after dCRT compared to patients with E-SCC [21], might partially explain the higher distant recurrence rate in the study by Haefner and colleagues [7], the reason for the higher rate of distant tumor recurrences in the study by Barbetta et al. [8] remains unclear. However, we have to point out, that the rate of distant recurrences in our study might be slightly underestimated. Due to the retrospective nature of our study, not all patients underwent periodic computed tomography during follow-up. While survival data of patients were completed by contacting the local registration offices, these data provided no further information about tumor recurrence. The use of different chemotherapy regimens (cisplatin/ 5-fluorouracil or carboplatin and paclitaxel) in our study should not affect results, because two retrospective trials did show significant differences regarding oncologic outcome between those two regimens for E-SCC patients undergoing dCRT or nCRT $+\mathrm{S}[22,23]$.

As it was mentioned before, our study has some limitations. One important limitation of this study is its retrospective nature. Also the moderate imbalances regarding tumor parameters between the patient cohorts (rate of lymph node metastases and tumor location) might affect the results and should be kept in mind. However, we also want to mention some strengths of our study. We only included patients with a curative treatment approach and who received complete treatment. That means that patients in the $\mathrm{nCRT}+\mathrm{S}$ group had to receive at least $41.4 \mathrm{~Gy}$ radiation dose and patients within the dCRT group underwent radiation therapy with at least $50 \mathrm{~Gy}$. In addition, all patients received simultaneous chemotherapy.

\section{Conclusion}

In E-SCC patients treated with either dCRT or nCRT $+\mathrm{S}$, a higher rate of local tumor recurrence was seen in patients treated with dCRT than in patients treated with nCRT + S. There was at least a trend towards an improved OS and PFS in patients undergoing nCRT $+\mathrm{S}$. However, this should be interpreted with caution, because proximal tumor location was the only parameter independently affecting the risk of local tumor recurrence.

\footnotetext{
Abbreviations

3D-CRT: 3-dimensional conformal radiotherapy; 5FU: 5-fluoruracil; AC: Adenocarcinoma; CRT: Chemoradiation; CT: Computed tomography; dCRT: Definitive chemoradiation; DFS: Disease-free survival; EC: Esophageal cancer; E-SCC: Esophageal squamous cell carcinoma; ${ }^{18}$ FDG-PET/ $\mathrm{CT}:{ }^{18}$ Fludeoxyglucose positron emission tomography with computed tomography; GTV: Gross tumor volume; Gy: Gray; IMRT: Intensity-modulated radiotherapy; nCRT + S: Neoadjuvant chemoradiation followed by surgery; OS: Overall survival; PFS: Progression-free survival; PTV: Planning target volume
}

\section{Acknowledgements}

Not applicable.

\section{Funding}

This research did not receive any specific grant from funding agencies in the public, commercial, or not-for-profit sectors.

\section{Availability of data and materials}

The datasets used and/or analysed during the current study are available from the corresponding author on reasonable request.

\section{Authors' contributions}

SM developed the study design, collected, and interpreted data, performed statistical analysis and drafted the manuscript. SP and MD made substantial contributions to conception and design of the study, interpreted data and revised the manuscript. HD, MF, HF, WW, MJ, RB and SC contributed significantly to the discussion and interpretation of the results. DH made substantial contributions to conception and design of the study, analyzed and interpreted data and revised the manuscript. All authors read and approved the final manuscript.

\section{Ethics approval and consent to participate}

The study was performed in accordance with the ethics standards at the Technical University of Munich (TUM) (ethical vote $N^{\circ} 250 / 18 S$ ).

\section{Consent for publication}

Not applicable.

\section{Competing interests}

The authors declare that they have no competing interests.

\section{Publisher's Note}

Springer Nature remains neutral with regard to jurisdictional claims in published maps and institutional affiliations.

\section{Author details}

${ }^{1}$ Department of Radiation Oncology, Klinikum rechts der Isar, Technical University Munich, Ismaninger Str. 22, 81675 Munich, Germany. ${ }^{2}$ German Cancer Consortium (DKTK), Partner Site Munich, Munich, Germany. ${ }^{3}$ Department of Surgery, Klinikum rechts der Isar, Technical University Munich, Ismaninger Str. 22, 81675 Munich, Germany. Institute of Pathology, Klinikum rechts der Isar, Technical University Munich, Ismaninger Str. 22, 81675 Munich, Germany. ${ }^{5}$ Institute of Radiology, Klinikum rechts der Isar, Technical University Munich, Ismaninger Str. 22, 81675 Munich, Germany. ${ }^{6} \mathrm{Helmholtz} \mathrm{Zentrum} \mathrm{München,} \mathrm{Institute} \mathrm{of} \mathrm{Radiation} \mathrm{Medicine} \mathrm{(IRM),}$ Ingolstädter Landstraße 1, 85764 Oberschleißheim, Germany.

Received: 19 October 2018 Accepted: 5 April 2019

Published online: 16 April 2019

\section{References}

1. Tepper J, et al. Phase III trial of trimodality therapy with cisplatin, fluorouracil, radiotherapy, and surgery compared with surgery alone for esophageal cancer: CALGB 9781. J Clin Oncol. 2008;26(7):1086-92.

2. van Hagen $\mathrm{P}$, et al. Preoperative chemoradiotherapy for esophageal or junctional cancer. N Engl J Med. 2012;366(22):2074-84.

3. Sjoquist KM, et al. Survival after neoadjuvant chemotherapy or chemoradiotherapy for resectable oesophageal carcinoma: an updated meta-analysis. Lancet Oncol. 2011;12(7):681-92.

4. National Comprehensive Cancer Network. Clinical practise guidelines in oncology - esophageal and esophagogastric junction cancers. (2018) https://www.nccn.org/professionals/physician_gls/pdf/esophageal.pdf. Accessed 02 June 2018.

5. Stahl M, et al. Chemoradiation with and without surgery in patients with locally advanced squamous cell carcinoma of the esophagus. J Clin Oncol. 2005;23(10):2310-7.

6. Bedenne L, et al. Chemoradiation followed by surgery compared with chemoradiation alone in squamous cancer of the esophagus: FFCD 9102. J Clin Oncol. 2007;25(10):1160-8. 
7. Haefner MF, et al. Neoadjuvant versus definitive chemoradiotherapy for locally advanced esophageal cancer : outcomes and patterns of failure. Strahlenther Onkol. 2018;194(2):116-24.

8. Barbetta A, et al. Definitive chemoradiotherapy versus neoadjuvant chemoradiotherapy followed by surgery for stage II to III esophageal squamous cell carcinoma. J Thorac Cardiovasc Surg. 2018;155(6):2710-21.e3.

9. Hategan $\mathrm{M}$, et al. Trimodality therapy and definitive chemoradiotherapy for esophageal cancer: a single-center experience and review of the literature. Dis Esophagus. 2015;28(7):612-8.

10. Liao Z, et al. Esophagectomy after concurrent chemoradiotherapy improves locoregional control in clinical stage II or III esophageal cancer patients. Int J Radiat Oncol Biol Phys. 2004;60(5):1484-93.

11. Nomura M, et al. Comparison between surgery and definitive chemoradiotherapy for patients with resectable esophageal squamous cell carcinoma: a propensity score analysis. Int J Clin Oncol. 2016;21(5):890-8.

12. Munch $\mathrm{S}$, et al. Comparison of dosimetric parameters and toxicity in esophageal cancer patients undergoing 3D conformal radiotherapy or VMAT. Strahlenther Onkol. 2016;192(10):722-9.

13. Haefner MF, et al. Intensity-modulated versus 3-dimensional conformal radiotherapy in the definitive treatment of esophageal cancer: comparison of outcomes and acute toxicity. Radiat Oncol. 2017;12(1):131.

14. Becker $\mathrm{K}$, et al. Histomorphology and grading of regression in gastric carcinoma treated with neoadjuvant chemotherapy. Cancer. 2003;98(7):1521-30.

15. Shi $\mathrm{H}$, et al. Does tumour location influence postoperative long-term survival in patients with oesophageal squamous cell carcinoma? Eur J Cardiothorac Surg. 2015;48(2):266-72.

16. Lloyd S, Chang BW. Current strategies in chemoradiation for esophageal cancer. J Gastrointest Oncol. 2014;5(3):156-65.

17. Kayani B, et al. Lymph node metastases and prognosis in oesophageal carcinoma--a systematic review. Eur J Surg Oncol. 2011;37(9):747-53.

18. Wilson $\mathrm{M}$, et al. Prognostic significance of lymph node metastases and ratio in esophageal cancer. J Surg Res. 2008;146(1):11-5.

19. Akutsu $Y$, et al. The number of pathologic lymph nodes involved is still a significant prognostic factor even after neoadjuvant chemoradiotherapy in esophageal squamous cell carcinoma. J Surg Oncol. 2012;105(8):756-60.

20. Robb WB, et al. Patterns of recurrence in early-stage oesophageal cancer after chemoradiotherapy and surgery compared with surgery alone. Br J Surg. 2016;103(1):117-25.

21. Xi M, et al. The impact of histology on recurrence patterns in esophageal cancer treated with definitive chemoradiotherapy. Radiother Oncol. 2017;124(2):318-24.

22. Munch $\mathrm{S}$, et al. Comparison of neoadjuvant chemoradiation with carboplatin/ paclitaxel or cisplatin/5-fluoruracil in patients with squamous cell carcinoma of the esophagus. Radiat Oncol. 2017;12(1):182.

23. Munch S, et al. Comparison of definite chemoradiation therapy with carboplatin/paclitaxel or cisplatin/5-fluoruracil in patients with squamous cell carcinoma of the esophagus. Radiat Oncol. 2018;13(1):139.

Ready to submit your research? Choose BMC and benefit from:

- fast, convenient online submission

- thorough peer review by experienced researchers in your field

- rapid publication on acceptance

- support for research data, including large and complex data types

- gold Open Access which fosters wider collaboration and increased citations

- maximum visibility for your research: over $100 \mathrm{M}$ website views per year

At $\mathrm{BMC}$, research is always in progress.

Learn more biomedcentral.com/submissions 